\title{
Postlaminectomy cervical deformity
}

\author{
Harel Deutsch, M.D., Regis W. Haid, M.D., Gerald E. Rodts, M.D., \\ and Praveen V. Mummaneni, M.D.
}

Chicago Institute of Neurosurgery and Neuroresearch, Chicago, Illinois; and Department of Neurosurgery, The NeuroSpine Institute, Emory University, Atlanta, Georgia

\begin{abstract}
Postlaminectomy cervical kyphosis is an important consideration when performing surgery. Identifying factors predisposing to postoperative deformity is essential. The goal is to prevent postlaminectomy cervical kyphosis while exposing the patient to minimal additional morbidity. When postlaminectomy kyphosis does occur, surgical correction is often required and performed via an anterior, posterior, or combined approach. The authors discuss the indications for surgical approaches as well as clinical results.
\end{abstract}

\section{KeY WORDS • postlaminectomy • deformity • kyphosis • cervical spine}

Performing extensive multilevel laminectomies typically does not immediately destabilize an otherwise intact spine. Removal of the posterior tension band, however, can result in progressively increasing compressive loads on the anterior $\mathrm{VB}$, resulting in anterior wedge compression. Surgery results in denervation and atrophy of the posterior cervical muscles. Additionally, surgery denervates and disturbs the facet joints. Loss of the posterior tension band increases the force on the anterior VB, worsens the sagittal deformity, and results in a kyphotic angulation (Fig. 1). As the kyphotic deformity progresses, the spinal cord may become progressively draped over the posterior VB, resulting in flattening of small feeding vessels to the cord as well as myelopathy. ${ }^{4}$ In addition to neurological dysfunction, patients may experience severe mechanical neck pain secondary to facet joint disruption. Postlaminectomy spinal deformity is an important consideration when performing surgery.

There are extensive data concerning the incidence of kyphotic deformity following laminectomy for cervical spondylotic myelopathy. The incidence of postoperative kyphosis after multilevel cervical spine laminectomy is

Abbreviations used in this paper: $\mathrm{ACP}=$ anterior cervical plate; $\mathrm{CSM}=$ cervical spondylitic myelopathy; $\mathrm{VB}=$ vertebral body. approximately $20 \% .^{13}$ The incidence increases significantly in patients with preoperative cervical spine straightening.

The data concerning postoperative cervical kyphosis after resection of spinal cord glioma are not as complete. The two patient groups differ significantly in their spinerelated biomechanics. Patients with CSM are older, and on many occasions their cervical spines have been partially fused, which are more stable than those in younger patients with nonarthritic spinal cord glioma. Thus, in younger patients undergoing cervical laminectomies for spinal tumors, the incidence rate of postoperative kyphosis may be higher than the $20 \%$ documented in elderly patients with CSM. ${ }^{3}$

The biomechanical characteristics of the cervical spine make it particularly prone to a postlaminectomy deformity. Analysis of biomechanical data indicates that the anterior VB supports only $36 \%$ of cervical load compared with $64 \%$ in the posterior column. ${ }^{20,22}$ Therefore, posterior facet joint disruption has a significant impact on cervical spine biomechanics. In children the incidence of postlaminectomy kyphotic spinal deformity is higher. ${ }^{6,28}$ Because the incompletely ossified VBs in children offer poor resistance to compressive forces, they are more prone to developing wedge deformity and progressive loss of sagittal balance. ${ }^{28}$ 


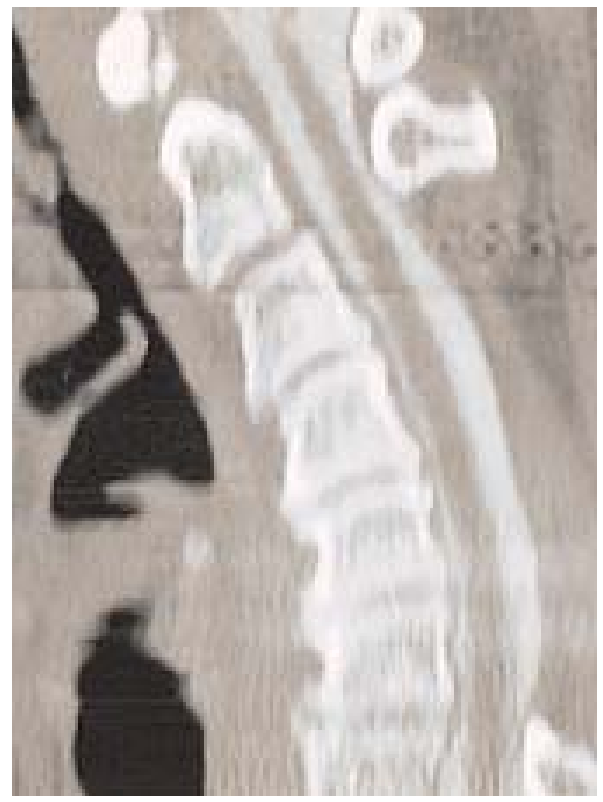

Fig. 1. Sagittal CT reconstruction demonstrating a postlaminectomy kyphosis.

Several factors have been associated with a higher incidence of postoperative kyphosis such as preoperative loss of cervical lordosis, facet capsule destruction, tumor, and irradiation. In several studies preoperative alignment has been shown to be an important predictor of postoperative deformity. Preoperative loss of lordosis is associated with a twofold increase in the incidence of postoperative kyphosis $^{13}$ (Fig. 2). Other factors implicated in an increased risk of postoperative kyphosis include facet joint capsule destruction and the extent of laminectomies. ${ }^{14,17,21,29}$ Poor postoperative neurological status has been implicated in worsening postoperative deformity, but no conclusive evidence exists.

Irradiation has been associated with higher postoperative deformity rates in children. Radiation causes bone death and impaired bone growth resulting in delayed deformity in growing children. Doses exceeding 3000 rads have been linked to higher rates of scoliosis. ${ }^{19}$ Mayfield, et al., ${ }^{19}$ reviewed data obtained in 74 patients who underwent radiotherapy for neuroblastoma in whom survival was greater than 5 years; they reported a $76 \%$ incidence of spinal deformity after a 13-year follow up. Additionally, $20 \%$ of the survivors required deformity correction surgery. King and Stowe ${ }^{16}$ documented 13 spinal fusions in 333 patients in whom radiotherapy was performed for either Wilm tumor or neuroblastoma. Spinal deformity is associated with increasing radiation doses. ${ }^{16}$

\section{CLINICAL PRESENTATION AND RADIOLOGICAL CHARACTERISTICS}

The most frequent presenting complaint is back or neck pain. The cause of the pain may be multifactorial. Causes in this population include muscle fatigue, facet joint arthropathy, or radiculopathy due to foraminal compression. Neurological sequelae such as myelopathy are late

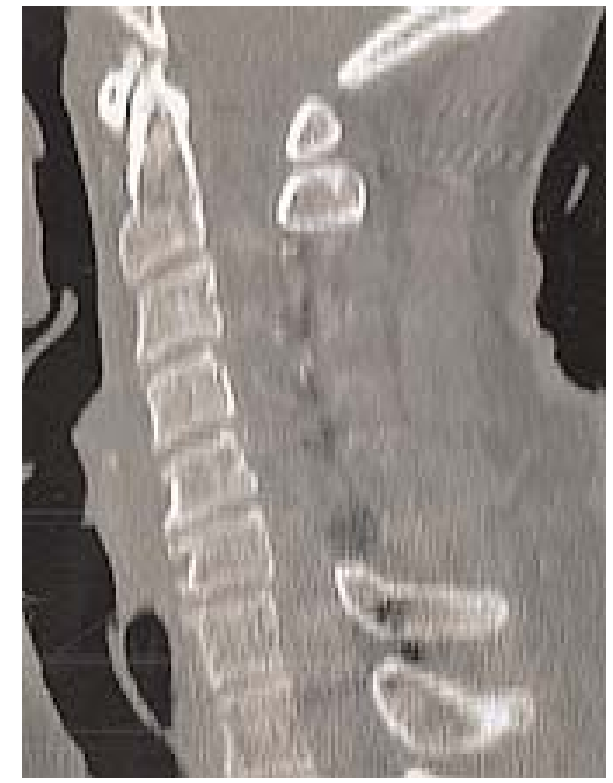

Fig. 2. Preoperative sagittal CT reconstruction demonstrating cervical straightening with a loss of normal cervical lordosis.

changes. Patients should undergo careful examination to determine if myelopathy is present. Lhermite sign can also be observed in some patients.

For patients presenting with pain, the surgeon must deduce its cause by evaluating the patient's history, examination findings, and/or diagnostic testing data. Fatiguerelated pain worsens as the day progresses and resolves with rest. Fatigue-related back pain is often alleviated by physical therapy (neck-muscle strengthening). If physical therapy fails, then brace therapy may be an option to reduce the workload on the muscles.

The cervical spine has a mean lordotic curve of 14 to $20^{\circ} .^{7,31}$ Any cervical kyphosis is abnormal. Evaluation includes standing anteroposterior and lateral radiography as well as MR imaging and CT scanning. Flexion-extension radiography is important in diagnosing any spondylolisthesis and curve-related flexibility.

\section{PREVENTION OF POSTOPERATIVE KYPHOTIC DEFORMITY}

The most prudent treatment involves initial deformity prevention. Several strategies have been used to prevent postoperative sagittal deformity after multilevel laminectomies.

One option involves posterior segmental instrumentation and fusion performed at the time of the initial surgery. Segmental instrumentation-augmented fusion adds considerable time and morbidity to the operation. ${ }^{8}$ Additionally, multilevel fusion will result in a substantial decrease in mobility. Placement of extensive instrumentation is also generally avoided in growing children with the highest sagittal deformity rates. Furthermore, levels adjacent to a long fusion will be subjected to larger stresses and may degenerate over time. Currently, segmental instrumentation is primarily considered in cases involving extensive bone removal or in those with other factors predisposing 
them to immediate postoperative instability. Patients with cervical kyphosis who must undergo a cervical laminectomy should also be considered candidates for posterior segmental instrumentation and lateral mass fusion.

Laminoplasty or osteoplastic laminotomy has been performed to prevent future cervical deformity. ${ }^{1,15}$ Laminoplasty has been well tolerated in cases of $\mathrm{SCM}^{8}$ and does not add significant operative time or morbidity. The lamina can be fixed in place using sutures, craniofacial plates and screws, or newly developed TiMesh LP laminoplasty instrumentation (Sofamor Danek, Memphis, TN) (Fig. 3). The lamina fuses to the posterior elements and reforms an arch. Laminoplasty has been shown to decrease postoperative deformity. ${ }^{11,29}$ Some authors, however, have reported the occurrence of deformity after laminoplasties. ${ }^{10,29}$

\section{TREATMENT OF POSTOPERATIVE KYPHOTIC DEFORMITY}

Once deformity has developed there are several surgical strategies available. Conservative treatment options may be chosen initially. Frequent radiographic follow-up evaluation and exercises are necessary. The role of brace therapy remains controversial.

Patients with progressive neurological decline, functional loss, or intractable pain are candidates for operative correction. The surgical strategy chosen depends largely on the pathological condition. If the deformity can be reduced, it can be corrected by careful positioning on the operating room table or in traction. The patient's spine can then be fused in the correct position via a posterior approach. ${ }^{5}$ Cranial traction for up to 5 days is also an option to reduce deformity. In the majority of cases the deformity cannot be reduced. For fixed deformity, anterior surgical release is necessary to correct the deformity. Placement of posterior instrumentation can further stabilize the spine and reduce the risk of the graft extrusion or breakage. ${ }^{9,30}$

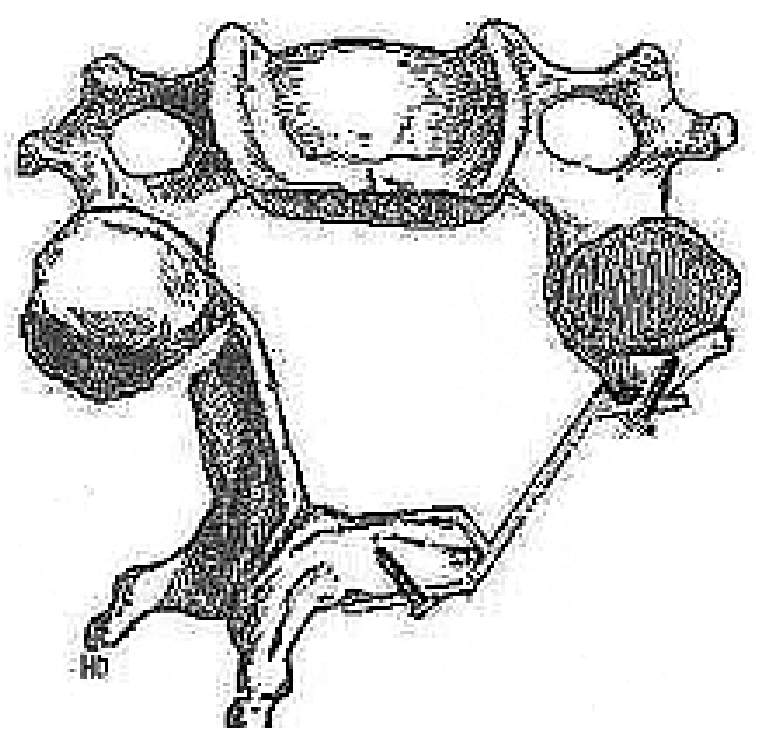

Fig. 3. Diagram illustrating the laminoplasty plate and screw system used to secure the lamina to the cervical lateral mass.

\section{Anterior Approach}

The cervical spine is generally amenable to deformity correction. The task of performing anterior and posterior approaches is straightforward. Significant correction can be achieved by anterior distraction and either discectomies or vertebrectomies. ${ }^{2,9}$ Herman and Sonntag 9 studied 20 patients with postlaminectomy kyphotsis in whom the mean angle was $38^{\circ}$. They found that traction improved the kyphotic angle by a mean of $8^{\circ}$. Open reduction via only an anterior approach and a vertebrectomy resulted in a mean improvement of $28^{\circ}$. Steinmetz, et al., ${ }^{26}$ reviewed a series of 10 patients with postlaminectomy deformity. Their mean kyphotic deformity was $13^{\circ}$. The mean correction was $20^{\circ}$ and the mean postoperative lordotic angle was $6^{\circ}$; both were achieved using only an anterior approach.

Whenever possible, we prefer to preserve the VBs and perform multiple discectomies and disc interspace distraction to restore the normal cervical lordosis. Allograft can be used, although in cases involving multilevel fusion and in patients who smoke, the placement of an anterior autograft is preferable. An ACP stabilizes the spine and increases fusion rates, especially in multilevel constructs. ${ }^{12}$ In early reports involving anterior correction without ACPs, the anterior construct failed in up to half of all cases. ${ }^{24,30}$ When vertebrectomy is necessary, an anterior fibula strut graft or an autograft-filled cage can be used.

More recently we have had excellent fusion rates using a polyetheretherketone interbody spacer in conjunction with recombinant human bone morphogenetic protein. Using either of these two materials avoids the morbidities associated with harvesting of iliac crest bone and achieves very high fusion rates even in cases with multiple levels (Fig. 4).

We generally prescribe a rigid cervical collar for 12 weeks after surgery in all cases of deformity surgery.

\section{Posterior Approach}

Posterior cervical fixation is achieved involving seg-
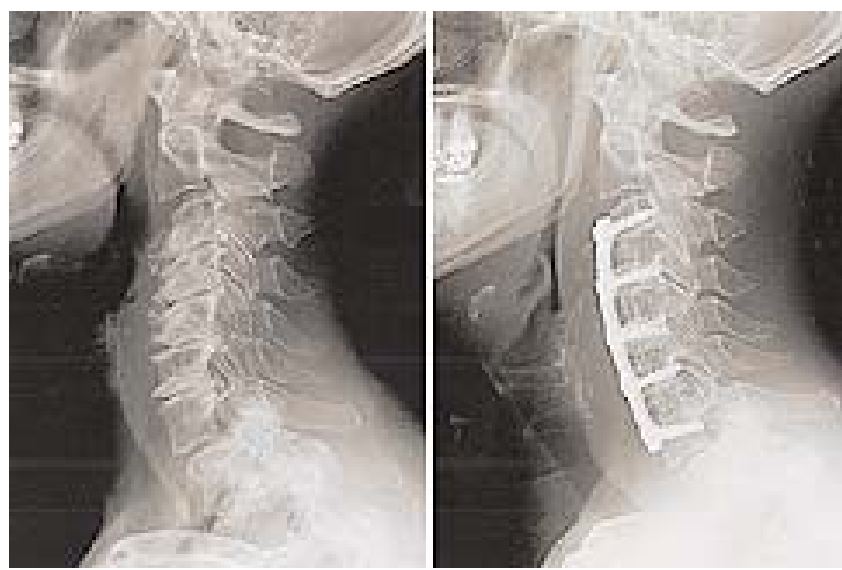

Fig. 4. Lateral radiographs. Left: Preoperative radiograph revealing degenerative cervical kyphosis. Right: Postoperative radiograph demonstrating excellent kyphotic correction with a fourlevel anterior cervical discectomy in which polyetheretherketone interbody spacers and bone morphogenetic protein were placed. 
mental lateral mass instrumentation with a polyaxial screw and rod system. Posterior instrumentation is required to supplement long strut grafts and relatively unstable cervical spines. Screw purchase on the lateral mass is limited to $16 \mathrm{~mm}$ or less, but for longer constructs such as in the upper thoracic pedicles, C-7, C-2, and C-1 lateral mass pedicle screws can be used (Fig. 5).

The amount of force that can be applied for deformity reduction is restricted by the limited screw purchase. Posterior osteotomies facilitate kyphotic deformity reduction. A limited degree of correction can be achieved by performing a Smith-Petersen osteotomy in which the posterior elements are excised (Fig. 6 upper).

Pedicle subtraction osteotomies can achieve a greater reduction but they have been associated with a high risk of neurological deterioration including quadriparesis and C-8 nerve root injury (Fig. 6 lower). Therefore, their use has been generally limited to extremely severe cases in which an anterior approach is not possible. Most reports in the literature are related to ankylosing spondylitis. ${ }^{25,27} \mathrm{Mc}-$ Master ${ }^{18}$ has reported performing osteotomies at C7- T1 in 15 patients in whom the mean correction was $54^{\circ}$; new neurological deficits developed in $20 \%$ of the patients.

\section{Anterior-Posterior Approach}

There is also support for a combined anterior-posterior arthrodesis, especially in cases in which the anterior procedure involves more than a two-level corpectomy. ${ }^{2,23}$ The anterior approach allows for deformity correction and the posterior approach supplements the construct stability. An anterior-posterior approach is also indicated in patients with an especially unstable spine. This applies to many postlaminectomy patients because their posterior tension band has already sustained significant compromise.

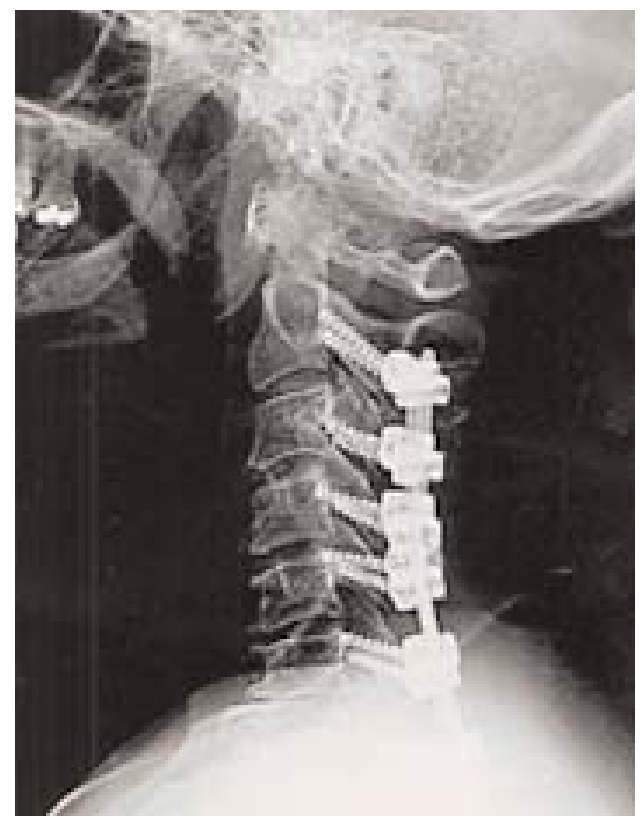

Fig. 5. Lateral radiograph demonstrating lateral mass screws and bilateral pars screws placed at C-2.

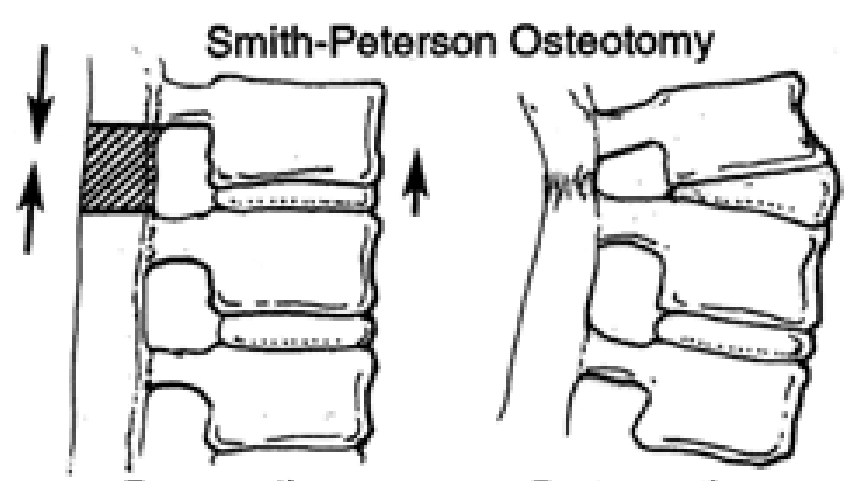

Postoperative

Whn ater Briduell

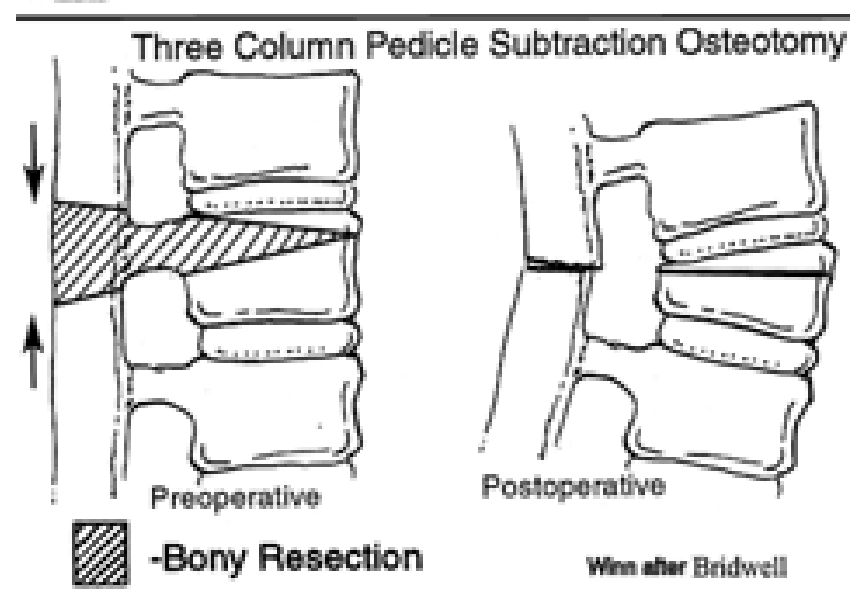

Fig. 6. Diagrams. Upper: Smith-Petersen osteotomy involves the removal of the posterior elements and can be used for reduction via a posterior approach. Lower: Pedicle subtraction osteotomy includes removal of the posterior elements, pedicle, and part of the VB.

\section{CONCLUSIONS}

Postlaminectomy spinal deformity is an important consideration in preoperative surgical planning. In young children and patients with preoperative sagittal deformity undergoing multilevel cervical and thoracic laminectomies, preventive measures are especially prudent. Performing a laminoplasty significantly helps to prevent postoperative spinal deformity. Posterior segmental instrumentation may be appropriate when a significant amount of bone work is necessary or if preoperative loss of lordosis is present. Postoperative deformity requiring surgery is usually treated via an anterior approach with ACP-assisted fusion. Extensive anterior operations may require posterior stabilization.

\section{References}

1. Abbott R, Feldstein N, Wisoff JH, et al: Osteoplastic laminotomy in children. Pediatr Neurosurg 18:153-156, 1992

2. Albert TJ, Vacarro A: Postlaminectomy kyphosis. Spine 23: m2738-2745, 1998

3. Bell DF, Walker JL, O’Connor G, et al: Spinal deformity after multiple-level cervical laminectomy in children. Spine 19: 406-411, 1994 
4. Breig A, el-Nadi AF: Biomechanics of the cervical spinal cord. Relief of contact pressure on and overstretching of the spinal cord. Acta Radiol Diagn 4:602-624, 1966

5. Callahan RA, Johnson RM, Margolis RN: Cervical facet fusion for control of instability following laminectomy. J Bone Joint Surg Am 59:991-1002, 1977

6. Cattell HS, Clark GL Jr: Cervical kyphosis and instability following multiple laminectomies in children. J Bone Joint Surg Am 49:713-720, 1967

7. Gore DR, Sepic SB, Gardner GM: Roentgenographic findings of the cervical spine in asymptomatic people. Spine 11: 521-524, 1986

8. Heller JG, Edwards CC Jr, Murakami H, et al: Laminoplasty versus laminectomy and fusion for multilevel cervical myelopathy: an independent matched cohort analysis. Spine 26: 1330-1336, 2001

9. Herman JM, Sonntag VK: Cervical corpectomy and plate fixation for postlaminectomy kyphosis. J Neurosurg 80:963-970 , 1994

10. Hosalkar HS, Pill SG, Sun PP, et al: Progressive spinal lordosis after laminoplasty in a child with thoracic neuroblastoma. J Spinal Disord Tech 15:79-83, 2002

11. Inoue A, Ikata T, Katoh S: Spinal deformity following surgery for spinal cord tumors and tumorous lesions: analysis based on an assessment of the spinal functional curve. Spinal Cord 34:536-542, 1996

12. Kaiser MG, Haid RW Jr, Subach BR, et al: Anterior cervical plating enhances arthrodesis after discectomy and fusion with cortical allograft. Neurosurgery 50:229-238, 2002

13. Kaptain GJ, Simmons NE, Replogle RE, et al: Incidence and outcome of kyphotic deformity following laminectomy for cervical spondylotic myelopathy. J Neurosurg (Spine 2) 93: 199-204, 2000

14. Katsumi Y, Honma T, Nakamura T: Analysis of cervical instability resulting from laminectomies for removal of spinal cord tumor. Spine 14:1171-1176, 1989

15. Kehrli P, Bergamaschi R, Maitrot D: Open-door laminoplasty in pediatric spinal neurosurgery. Childs Nerv Syst 12: 551-552, 1996

16. King J, Stowe S: Results of spinal fusion for radiation scoliosis. Spine 7:574-585, 1982

17. McLaughlin MR, Wahlig JB, Pollak IF: Incidence of postlaminectomy kyphosis after Chiari decompression. Spine 22: 613-617, 1997

18. McMaster MJ: Osteotomy of the cervical spine in ankylosing spondylitis. J Bone Joint Surg Br 79:197-203, 1997
19. Mayfield JK, Riseborough EJ, Jaffe N, et al: Spinal deformity in children treated for neuroblastoma. J Bone Joint Surg Am 63:183-193, 1981

20. Pal GP, Sherk HH: The vertical stability of the cervical spine. Spine 13:447-449, 1988

21. Papagelopoulos PJ, Peterson HA, Ebersold MJ, et al: Spinal column deformity and instability after lumbar or thoracolumbar laminectomy for intraspinal tumors in children and young adults. Spine 22:442-451, 1997

22. Raynor RB, Pugh J, Shapiro I: Cervical facetectomy and its effect on spine strength. J Neurosurg 63:278-282, 1985

23. Sasso RC, Ruggiero RA Jr, Reilly TM, et al: Early reconstruction failures after multilevel cervical corpectomy. Spine 28: 140-142, 2003

24. Sim FH, Svien HJ, Bickel WH, et al: Swan-neck deformity following extensive cervical laminectomy. A review of twentyone cases. J Bone Joint Surg Am 56:564-580, 1974

25. Simmons EH: Kyphotic deformity of the spine in ankylosing spondylitis. Clin Orthop 128:65-77, 1977

26. Steinmetz MP, Kager CD, Benzel EC: Ventral correction of postsurgical cervical kyphosis. J Neurosurg (Spine 1) 98:1-7, 2003

27. Van Royen BJ, De Gast A, Smit TH: Deformity planning for sagittal plane corrective osteotomies of the spine in ankylosing spondylitis. Eur Spine J 9:492-498, 2000

28. Yasuoka S, Peterson HA, Laws ER Jr, et al: Pathogenesis and prophylaxis of postlaminectomy deformity of the spine after multiple level laminectomy: Difference between children and adults. Neurosurgery 9:145-152, 1981

29. Yeh JS, Sgouros S, Walsh AR, et al: Spinal sagittal malalignment following surgery for primary intramedullary tumours in children. Pediatr Neurosurg 35:318-324, 2001

30. Zdeblick TA, Bohlman HH: Cervical kyphosis and myelopathy. Treatment by anterior corpectomy and strut-grafting. J Bone Joint Surg Am 71:170-182, 1989

31. Zdeblick TA, Zou D, Warden KE, et al: Cervical stability after foraminotomy. A biomechanical in vitro analysis. J Bone Joint Surg Am 74:22-27, 1992

Manuscript received July 28, 2003.

Accepted in final form August 17, 2003.

Address reprint requests to: Harel Deutsch, M.D., Department of Neurosurgery, Rush-Presbyterian-St. Luke's Medical Center, 1725 Harrison Street Suite 970, Chicago, Illinois 60612. email: hdeutsch@cinn.org. 